\title{
Decision factors affecting line orientation judgments in the method of single stimuli
}

\author{
RUFIN VOGELS and GUY A. ORBAN \\ Katholieke Universiteit Leuven, Leuven, Belgium
}

\begin{abstract}
Just noticeable differences in orientation are smaller at principal standard orientations than at oblique standard orientations when they are measured with the method of single stimuli. We determined whether this oblique effect is due to an anisotropy in decision factors. A first series of experiments showed that the subjects compare the stimulus with an internal criterion, and that this decision rule is used at all standard orientations. A second series of experiments determined the influence on the oblique effect of nonsensorial variables related to criterion setting. The results strongly suggest that the effect is not due to a criterial noise anisotropy and that criterion-setting processes are similar at principal and oblique standard orientations. The latter conclusion was also supported by an analysis of the sequential stimulus and response dependencies in this task. Hence, it appears that the oblique effect in line orientation discrimination, when it is measured with the method of single stimuli, is due not to decision factors but to a sensorially based anisotropy.
\end{abstract}

It is now well established that line orientation discrimination shows an oblique effect: Just noticeable differences (JNDs) in orientation are smaller at horizontal and vertical standard orientations than at oblique standard orientations (Matin \& Drivas, 1979; Orban, Vandenbussche, \& Vogels, 1984; Vogels, Orban, \& Vandenbussche, 1984). It has been proposed that this oblique effect is a consequence of the fact that more S-cells in area 17 are tuned to horizontal and vertical stimulus orientations than to oblique stimulus orientations (Kennedy, Martin, Orban, \& Whitteridge, 1985; Orban \& Kennedy, 1981; Orban et al., 1984). Indeed, the pattern of the meridional variations in the number of S-cells of the baboon's striate cortex closely matches the pattern of meridional variations in line orientation discrimination (Orban et al., 1984). Also, properties of the oblique effect in orientation discrimination, such as its line length dependence (Orban et al., 1984) and variation with eccentricity (Vandenbussche, Vogels, \& Orban, 1986), could be predicted from the properties of the S-cells as measured in physiological studies.

Although these findings support the hypothesis linking the orientation anisotropy of the S-cells and line orientation discrimination, they do not prove it. In particular, it is necessary to show that this oblique effect is not due to an anisotropy in the decision process but instead results from a sensorially based anisotropy. We chose to investigate the role of nonsensorial factors in the oblique ef-

The authors thank S. McKee for a critical reading of an earlier draft of this paper. We are grateful to $M$. Van Lammeren (Department of Ophthalmology, K.U.L. Medical School) for performing the optometric examinations. The technical assistance of $P$. Kayenbergh, $G$. Vanparrijs, and G. Meulemans, as well as the expert typing of Y. Celis, is gratefully acknowledged.

The authors' address is: Laboratorium voor Neuro- en Psychofysiologie, KULeuven, Campus Gasthuisberg, Herestraat, B-3000 Leuven, Belgium. fect as measured by the method of single stimuli (MSS) since most of the parametric studies cited above were done with this paradigm (Orban et al., 1984; Vandenbussche et al., 1986; Vogels et al., 1984).

Results obtained with a modified absolute judgment paradigm (Matin, Drivas, \& Valle, 1982) suggest that part of the oblique effect could be due to nonsensorial factors such as criterial variance and vocabulary biases. However, it could well be that nonsensorial factors play a less important role when orientation sensitivity is determined with our MSS paradigm, since this procedure involves fewer stimulus and response alternatives than the one used by Matin et al. (1982). In our method the subject classifies five stimuli into two categories, each of which corresponds to one of two possible responses. These responses are associated with stimuli that are respectively smaller and larger than the median stimulus. The stimuli are presented sequentially and in random order. The major assumption behind this method is that the subject classifies these stimuli using an internal criterion (an absoluteidentification, or $\mathrm{AI}$, decision rule).

However, subjects could compare the stimulus on the present trial with the one on the preceding trial (a pairedcomparison, or PC, rule). We showed that the use of a particular decision rule, AI or PC, strongly affects the MSS thresholds by running a computer simulation using the MSS stimulus sequence with which we actually tested human subjects. It is obvious that the performance level of a subject using a PC rule depends on the mean difference between successively presented stimuli. We define the difference between two successively presented stimuli as the step size. We assumed the function describing the relation between the proportion of correct responses and the step size to be a normal ogive.

The results of the simulation are shown in Figure 1A. The PC rule yielded a threshold 3.6 times larger than the 

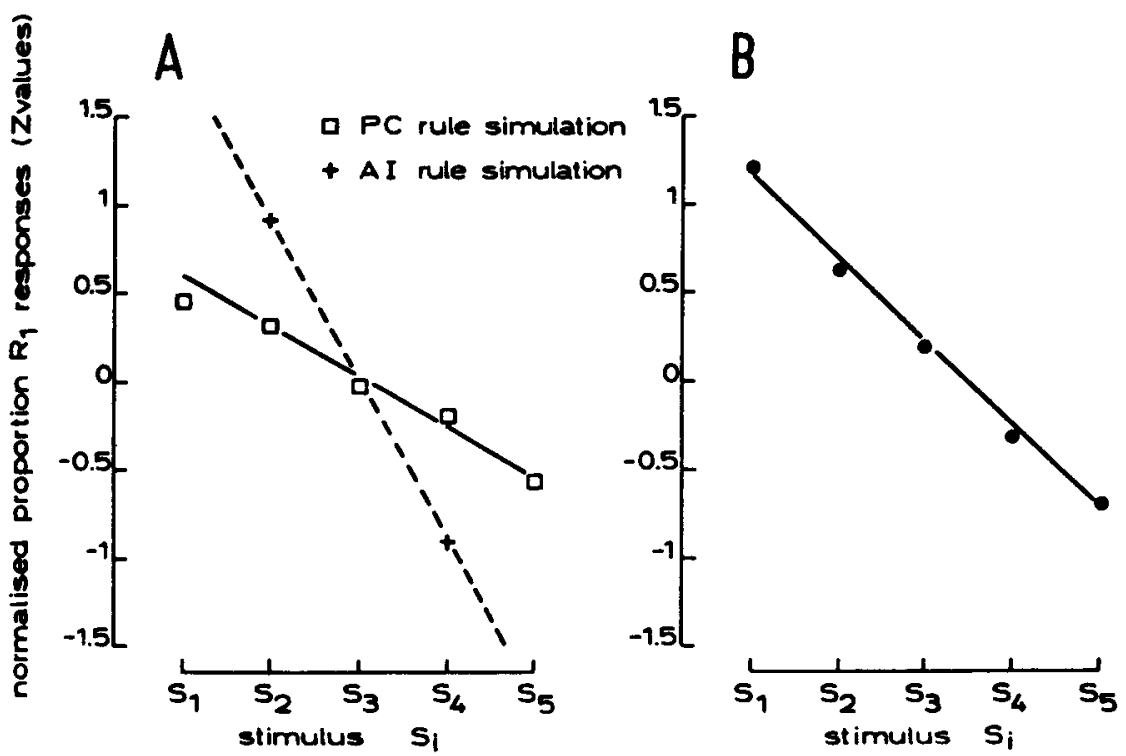

Figure 1. Effect of decision rule on performance in an MSS design. A. Results of a computer simulation. The proportion of $R_{1}$ responses was calculated as a function of the stimulus $S_{i}$, assuming the use of a PC rule (solid line) and an AI rule (dashed line). B. Results obtained with the same stimulus sequence at the horizontal standard orientation in a human sabject. The difference between $S_{1}$ and $S_{3}$ was $1^{\circ}$. The data are based on 1,600 responses.

one calculated assuming the use of an AI rule. This difference is similar to the magnitude of the oblique effect in line orientation discrimination (Vogels et al., 1984). Hence, the oblique effect could be due to an anisotropy of the decision rule used by the subjects. Furthermore, a comparison showed no reliable difference between either set of simulation data (Figure 1A) and data obtained from a human subject using the same stimulus sequence (Figure 1B). Thus the shape of an empirically obtained psychometric function cannot be used to differentiate between decision rules. However, by systematically manipulating the mean step size, one can find out whether a subject uses a PC rule, since, if he/she does, the size of the JND should be inversely related to mean step size.

Assuming an AI rule, other decision factors are related to the criterion used in an MSS task. We investigated whether (1) the buildup and (2) the maintenance of the criterion were different between standard orientations. We also compared the influence at principal and oblique standard orientations of factors such as feedback, previewing, and stimulus frequency distribution, which are believed to affect the criterial variance. It is important to investigate the latter variables since the oblique effect could be a result of criterial variance that is greater at the oblique than at the principal orientations. Criterial variance was further investigated by analyzing both stimulusand response-dependent sequential effects, since such criterial variance consists mainly of criterion shifts due to sequential effects (Treisman \& Williams, 1984). This part of criterial variance can be estimated by calculating the values of the criteria as a function of the preceding stimulus or response.

\section{GENERAL METHOD}

\section{Subjects}

Thirteen subjects (male and female) with normal or corrected vision participated in this study. Only 1 subject (R.V.) was aware of the aim of the experiment. At least two JNDs were measured with the MSS as training for each subject (Orban et al., 1984).

\section{Apparatus}

A red fluorescent bar $15^{\circ}$ of visual angle long and $0.23^{\circ}$ wide was illuminated with ultraviolet light in a black painted box. The line appeared in a dark frameless environment. The contrast $(\log \Delta \mathrm{I} / \mathrm{I})$ was 0.1 . Exposure time was shutter controlled and was set at $600 \mathrm{msec}$. Before the end of the stimulus presentation, the subject had to signal the stimulus orientation by depressing one of two response keys. The intertrial interval was set at $5 \mathrm{sec}$. All experiments were performed monocularly with the right eye. Further details can be found in Orban et al. (1984).

\section{Procedure}

In this section we will describe the general MSS procedure used in this study. The aspects of the task that were changed to enable us to study the decision factors are described in the Results section.

The threshold determination consisted of two steps, training and test (Orban et al., 1984). In the training step, two bars with orientations symmetrical with respect to the standard orientation were presented in random order in blocks of 20 trials. The difference between each bar's orientation and the standard orientation (which was not presented) is called $\Delta$. It is important to note that on each trial only one line was present. The smallest difference for which the subject got at least $90 \%$ correct was determined by changing $\Delta \mathrm{s}$ in steps of $0.5^{\circ}$ or $1^{\circ}$ in successive blocks. Feedback (as to whether the response was correct or incorrect) was given. The test step was an MSS task with five stimulus and two response alternatives. The smallest $\Delta s$ for which the subject reached the $90 \%$ correct criterion in the preceding step were split into two equal intervals. This was done at either side of the standard orientation. Hence 
the stimulus set consisted of five orientations $\left(s_{o}+\Delta s, s_{o}+\Delta s / 2\right.$, $s_{0}, s_{0}-\Delta s / 2, s_{o}-\Delta s$, so being the standard orientation). These five stimuli were presented sequentially in four test blocks of $\mathbf{8 0}$ trials each. In most experiments, each block consisted of the same random stimulus sequence but with a different (random) starting point and direction. A 5-min rest period was inserted between test blocks and between training and test steps.

The results of the MSS task were analyzed as follows: The data from the four test blocks were pooled and the proportion of rightkey responses was calculated for each stimulus orientation. After $Z$ normalization of these proportions, the standard deviation of the corresponding normal distribution was calculated with linear regression and taken as the JND in orientation. Hence, the thresholds were $84 \%$ correct JNDs. The JNDs were log transformed, since the standard error increases nearly proportionally to the absolute level of the JND. All results were analyzed with two types of analysis of variance (ANOVA) (Kirk, 1968). We used factorial block-design ANOVAs when only one JND in orientation for each subject was available. If more than one observation for each subject was present, we used factorial-design ANOVAs with the subject variable as a supplementary factor (see Kirk, 1968).

\section{RESULTS}

\section{The Decision Rule in an MSS Line}

\section{Orientation Task (Experiments 1 and 2)}

Using the PC simulation discussed in the introduction, we computed the difference in JND in orientation between a stimulus sequence with a large mean step size and one with a small mean step size. The results of this simulation are shown in Figure 2A. The three functions correspond to simulations with different percent correct values for the largest step size. These ranged from 0.90 to 0.995 , which is realistic, since we determined the largest step size in the training step in such a way that the subject got at least $90 \%$ correct (see Method section). The JNDs of the small step size sequence were 6 to 8 times larger than those of the large step size sequence (Figure 2A). Other simulations showed this ratio to be independent of the amount of response bias.

Hence, if the subjects used a PC rule in these MSS stimulus sequences, we should observe similar ratios between JNDs obtained with small and large step size stimulus sequences. To minimize the effect of intersession variability, we ran, within one threshold determination, two blocks with a small and two with a large step size. The order of the blocks was randomized between threshold determinations and subjects. We ran four determinations, each consisting of four test blocks of 80 trials, at a principal and at an oblique standard orientation for 4 naive subjects.

The pooled data are shown in Figure 2B. A threefactorial ANOVA (subjects $\times$ standard orientation $\times$ step size) yielded three significant main effects: standard orientation $[F(1,48)=90.36, p<.0005]$, step size $[F(1,48)$ $=31.15, p<.0005]$, and subjects $[F(3,48)=7.44, p<$ $.0005]$. Each subject showed an oblique effect (Figures 2A and 2B); however, the interaction between step size and standard orientation was not significant $[F(1,48)=1.52$, n.s. $]$. The average JNDs in orientation were a factor of 1.5 and 1.9 larger for the small than for the large step size at the principal and oblique standard orientations, respectively. This is much lower than the

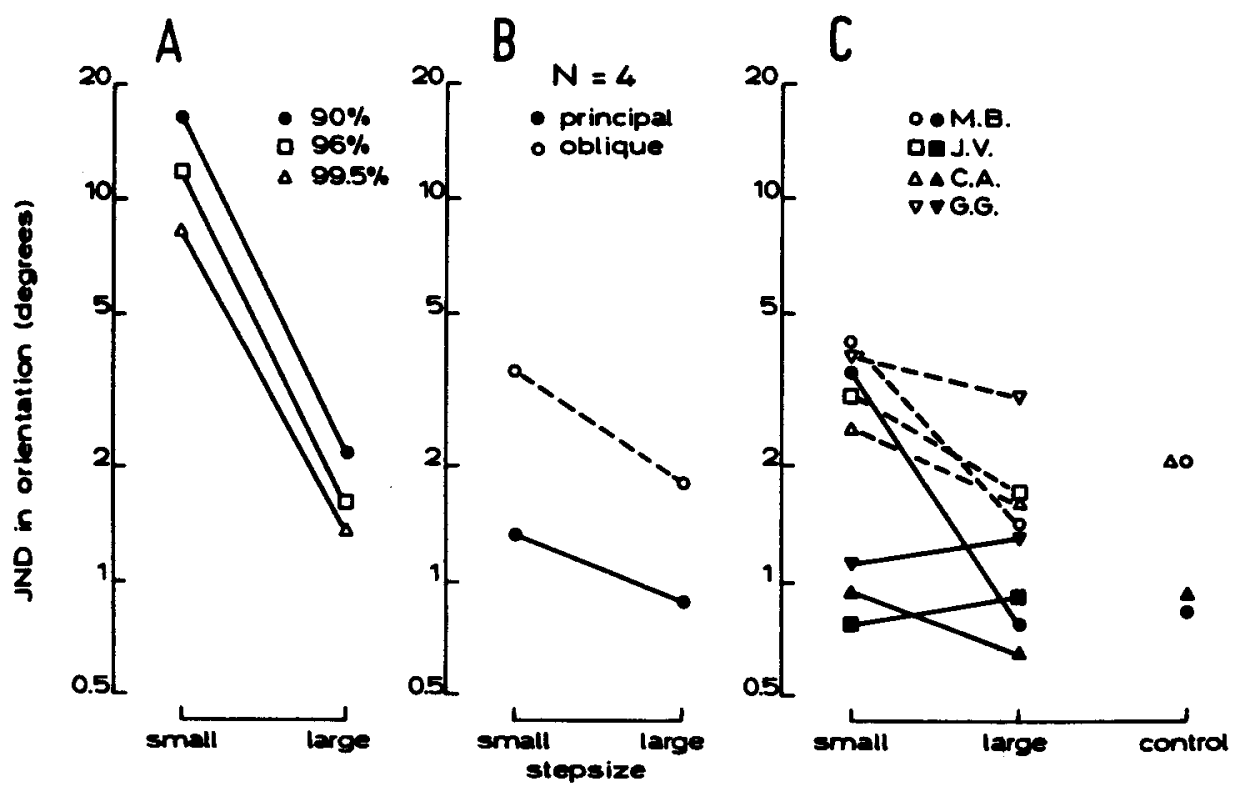

Figure 2. Effect of mean step size on orientation discrimination. A. Results of a computer simulation: The JND in orientation (in arbitrary units) as a function of the mean step size of the stimulus sequence. The proportion of correct responses for the largest stimulus difference was $90 \%, 96 \%$, or $99.5 \%$. B. The mean JND in orientation (for 4 subjects) as a function of mean step size at the principal (solid line) and oblique (dashed line) standard orientations. C. Individual data. The JNDs in orientation for the principal and oblique standard orientations are indicated by solid and open symbols, respectively. For 2 subjects, JNDs obtained with a stimulus sequence we usually use for testing (control) are also shown. 
ratio (6 to 8) expected under the assumption of a PC rule (Figure 2A). Since the interaction between the subject variable and the step size was strongly significant $[F(3,48)$ $=9.96, p<.0005$ ], we plotted the individual data in Figure 2C. The data of subject M.B. are somewhat similar to the PC simulation results (Figure 2A), especially for the principal standard orientation, although the observed ratio is still lower (3.7) than the one expected for a PC rule. For all other subjects, the data indicate that the $\mathrm{PC}$ rule is certainly not the rule used in the MSS task.

For 2 subjects, we also determined thresholds for the stimulus sequences that we use in our standard MSS task. These data are shown in Figure $2 \mathrm{C}$ (control data). If a subject used a PC rule, these JNDs should be a factor of 2 larger than those obtained for the large step size condition and a factor of 3.5 smaller than those obtained for the small step size condition. The observed average ratios (for 2 subjects) were $1.3( \pm .2)$ and $2.1(+1.2)$ (Figure 2C). Again, the data of subject M.B. are closest to the expected values.

These results strongly suggest that subjects use an AI rule in judging the orientation of lines in an MSS task. This hypothesis was further confirmed in an experiment in which we determined the effect of the number of stimulus alternatives in well-trained subjects. We compared threshold determinations with two, three, and five stimulus alternatives for 2 subjects (R.V. and B.D.B.). The stimulus difference $\Delta$ was constant across conditions. The test blocks consisted of 90 trials without feedback. Two standard orientations (horizontal and left oblique) were used. For each condition, two test blocks were run in an interleaved fashion.

The average JNDs were $0.7^{\circ}$ for two stimulus alternatives, $0.59^{\circ}$ for three stimulus alternatives, and $0.69^{\circ}$ for five stimulus alternatives at the horizontal standard orientation, and $0.98^{\circ}$ for two stimulus alternatives, $0.93^{\circ}$ for three stimulus alternatives, and $1.04^{\circ}$ for five stimulus alternatives at the oblique standard orientation. A threefactorial ANOVA (subjects $\times$ stimulus alternatives $\times$ standard orientation) yielded two significant main effects: subjects $[F(1,12)=15.95, p<.0005]$ and standard orientation $[F(1,12)=36.70, p<.0005]$. The number of stimulus alternatives showed no effect on the JNDs in orientation $[F(2,12)=1.56$, n.s. $]$. The interaction terms were not significant.

Because there is a negative correlation between the number of stimulus alternatives within a test block and the mean step size, these results confirm the inference drawn from the first experiment: Subjects do not use a PC rule in an MSS task. Furthermore, these results suggest that the oblique effect in line orientation discrimination assessed with the MSS is not due to a meridional difference in decision rule.

\section{The Buildup of the Criterion \\ in an MSS Task (Experiment 3)}

If a subject uses an AI rule, it is conceivable that the criterion used in the MSS test step is built up during the training step. To find out whether there is such a transfer from training to test step, we manipulated the time interval between them. The MSS performance of 2 subjects was measured for training-test intervals of $5 \mathrm{~min}, 3 \mathrm{~h}$, and $16 \mathrm{~h}$. We found no significant effect of the training-test interval at the principal or the oblique standard orientations, indicating that the criterion is built up during the test step itself. Thus, because the criterion has to be built up in the beginning of a test block, one should expect performance to be lower at the beginning than at the middle or end of an MSS test block. And one could argue that the time course of the buildup is different between standard orientations, which would explain the oblique effect. Hence, we determined five thresholds at an oblique and at a principal standard orientation for four subjects (J.V.P., H.S., S.P., and G.B.). These determinations were interleaved. For each subject and standard orientation we thus had 5 (determinations) $\times 4$ (test blocks per determination) $\times 80$ (trials in each test block) responses. Each test block was divided into eight subblocks of 10 trials. The data of the corresponding subblocks of the $5 \times 4$ test blocks were pooled. Hence, for each subject and standard orientation we could calculate JNDs for each of the eight pooled $(n=200)$ subblocks. To investigate whether the speed of the buildup of the criterion is affected by small step sizes, we determined five thresholds at each standard orientation, using a small step size, for 2 subjects (J.V.P. and S.P.). These results were pooled and analyzed in the same way as those of the normal step size sequence.

The JNDs for the normal step size sequences, averaged over 4 subjects, are shown in Figure $3 \mathrm{~A}$ as a function of the number of preceding subblocks within a test block. A two-factorial block-design (Kirk, 1968) ANOVA (standard orientation $\times$ subblock) indicated no significant effect of the subblock variable $[F(7,45)<1$, n.s. $]$. The oblique effect $[F(1,45)=293.2, p<.0005]$ was the only significant effect.

The results of the small step size sequence are shown in Figure 3B (squares). The average JNDs of the normal stimulus sequence obtained in these subjects are also shown (circles). A three-factorial block-design ANOVA (standard orientation $\times$ subblock $\times$ step size) was run on these data. Again, the subblock variable yielded no significant effect $[F(7,31)<1$, n.s.]. Both standard orientation $[F(1,31)=212.2, p<.0005]$ and step size $[F(1,31)=64.8, p<.0005]$ were significant. Also, the interaction between standard orientation and step size was significant $[F(1,31)=8.05, p<.01]$ : The step size effect was larger at oblique than at principal standard orientations. This suggests that in these subjects the establishment of a stable criterion in the small step size condition was more difficult at the oblique than at the principal standard orientation. Notice that there is a weak tendency for JNDs to decrease with time in the horizontal small step size condition. For the oblique small step size condition, the changes of the JNDs over time were more erratic.

It is important to note that for the normal stimulus sequence (Figure 3A) there is no significant interaction between standard orientation and subblock condition 

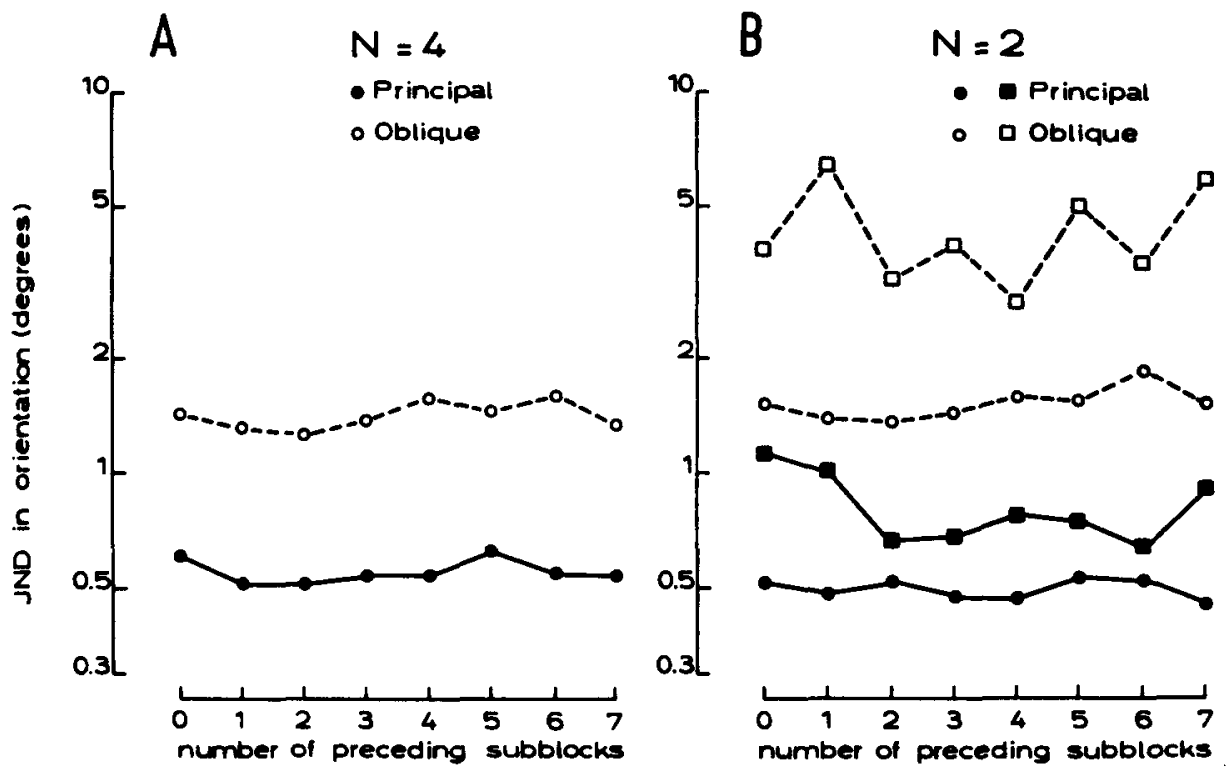

Figure 3. JNDs in orientation as a function of the number of preceding subblocks of 10 trials in an MSS test block of 80 trials. A. Mean JNDs (for 4 subjects) at the principal (solid symbols) and oblique (open symbols) standard orientations. B. Mean JNDs (for 2 subjects) for the normal step size (circles) and small step size (squares) conditions at the horizontal (solid symbols) and left oblique (open symbols) standard orientations.

$[F(7,45)<1$, n.s.], which suggests that the buildup of a criterion within a test block is highly similar at oblique and horizontal standard orientations. Therefore, buildup per se cannot explain the oblique effect measured with our standard MSS procedure.

\section{The Maintenance of the Criterion in an MSS Task (Experiment 4)}

If the criterion is built up very quickly and, once established, is available during the remainder of the test block, then one should expect that when one begins a test block with a normal randomized stimulus sequence and subsequently gives a small step size sequence, performance should not drop; that is, no step size effect should occur. If, however, performance drops, this indicates that optimal performance in an MSS task requires continuous, active maintenance of the criterion.

Hence, we determined thresholds with test blocks of 80 trials in which the stimulus sequences consisted of 20 trials of a normal (N) step size sequence followed by 60 trials of a small (S) step size sequence (NS condition). As a control, we ran a stimulus sequence consisting of 60 trials of a small step size sequence followed by 20 trials of a normal step size sequence (SN condition). To measure possible meridional differences in the criterion maintenance, we determined the thresholds in both conditions at a principal and at an oblique standard orientation. For each standard orientation, we determined six thresholds, each consisting of four test blocks of 80 trials, for 2 subjects (J.V.P. and I.H.). The two stimulus sequence conditions were interleaved within each threshold determination. Hence, each stimulus sequence condition was tested $6 \times 2$ times in each subject. Each test block was divided into four subblocks of 20 trials. The subblocks were averaged over threshold determinations, yielding two JNDs for each subblock, stimulus sequence condition, standard orientation, and subject. The average of the four JNDs (averaged within and between subjects) obtained for each combination of subblock, standard orientation, and stimulus sequence conditions is shown in Figure 4. The average JNDs obtained with a normal step size sequence are indicated by arrows. For both the SN condition and the NS condition we obtained a clear-cut effect of step size, which was already present in the first subblock following the change in mean step size.

The data were further analyzed using a four-factorial ANOVA (subjects $\times$ standard orientation $\times$ stimulus sequence condition $\times$ subblock). This analysis yielded three significant main effects: subjects $[F(1,32)=17.75, p<$ $.0005]$, standard orientation $[F(1,32)=148.9, p<$ $.0005]$, and subblocks $[F(3,32)=4.77, p<.01]$. The most interesting significant effect, however, is the interaction between the subblocks and the NS/SN variable $[F(3,32)=11.21, p<.0005]$. Inspection of Figure 4 indicates that the results of the NS condition are the mirror image of those of the SN condition. An ANOVA run on the same data, but with a reversed order of the subblocks of the SN sequence, confirms this observation: The interaction between the NS/SN variable and the subblock order was no longer significant $[F(3,32)<1]$. The three main effects remained significant in the latter analysis. The interaction between standard orientation and step size did not reach significance in either of the two ANOVAs $[F(3,32)<1$, n.s., in both analyses $]$. No interaction term 


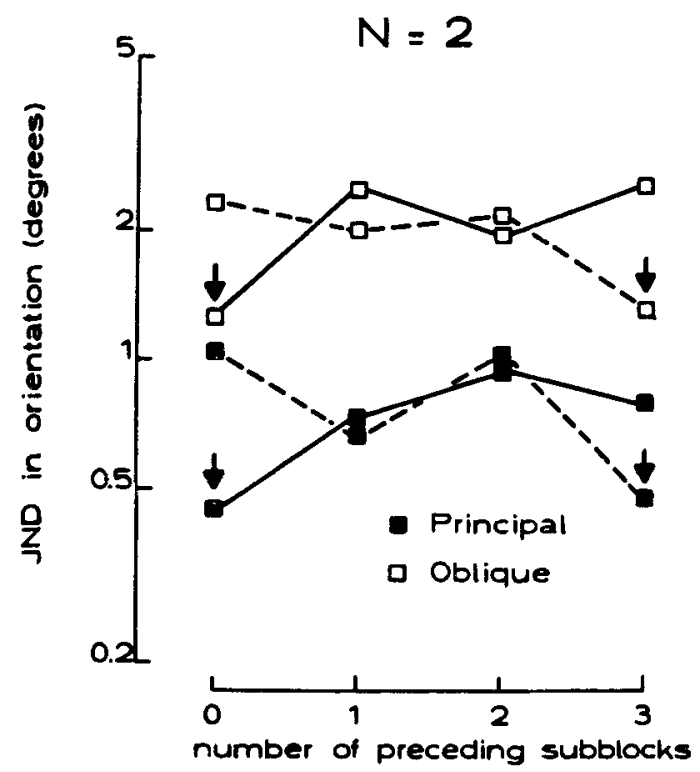

Figure 4. Effect of the number of preceding subblocks of 20 trials on the JNDs in orientation for two different stimulus sequence conditions (Experiment 8). In one stimulus sequence condition, a subblock with a normal step size was followed by three subblocks with a small step size (solid lines); in the other condition, three subblocks with a small step size were followed by one subblock with a normal step size (dashed lines). JNDs in orientation obtained in a subblock with a normal step size sequence are indicated by an arrow. Mean JNDs (for 2 subjects) are shown for the horizontal (solid symbols) and left oblique (open symbols) standard orientations.

containing the standard orientation variable and the subblock or NS/SN variable was significant in either of the two ANOVAs, indicating that these nonsensorial effects were similar for both standard orientations.

The results of this experiment suggest that active maintenance of the criterion is necessary during an MSS task. We also observed that the decay of performance with the decrease in mean step size is very fast (occurring during the first 20 trials after the change) (Figure 4). This holds for horizontal as well as for oblique standard orientations, suggesting that there is no meridional difference in the rate of decay of the performance due to a decrease in step size.

\section{The Effects of Previewing, Feedback,} and the Stimulus Frequency Distribution (Experiments 5, 6, and 7)

Previewing of the stimuli (Green \& Swets, 1966), stimulus feedback, or the frequency distribution of the stimuli may affect the criterial variance by influencing the setting of the criterion during the task. Hence, in Experiment 5 , we presented the two extreme stimuli three times in alternation before each test block (previewing condition). In Experiment 6, we told the subjects after each stimulus presentation which stimulus had been presented on that trial (feedback condition). In both experiments these threshold determinations were interleaved with determinations without either previewing or feedback. In
Experiment 7, thresholds were determined for stimulus sequences for which the stimulus frequency distributions are shown in Figure 5. In all of these experiments, two thresholds per condition were determined at a principal and at an oblique standard orientation for 2 subjects.

The mean JNDs in orientation for each subject and experimental condition are shown in Table 1 . In no experiment did we find a significant effect of the nonsensorial variable, nor were any of its interactions with the orientation variable significant. The latter fact indicates that none of these factors influence the oblique effect in the MSS task.

\section{Criterial Noise and Sequential \\ Dependencies in the MSS task}

Using the data from all experiments with a normal step size and without feedback or previewing, we calculated stimulus and response sequential dependencies for the principal and oblique standard orientations. We calculated the conditional proportions of left-key responses given stimulus $S_{i}$ or response $R_{i}$ on the preceding trial (Lag 1), or given $S_{i}$ or $R_{i}$ on the next preceding trial ( $\left.L a g 2\right)$, and so on. Then we constructed plots relating $Z\left[P\left(R_{2}\right)\right]$ to $S_{i}$ for each preceding $S_{i}$ and $R_{i}$ condition. Examples of such plots of stimulus dependencies are shown in Figure 6A for the five different $S_{i}$ in the preceding trial. The verti-
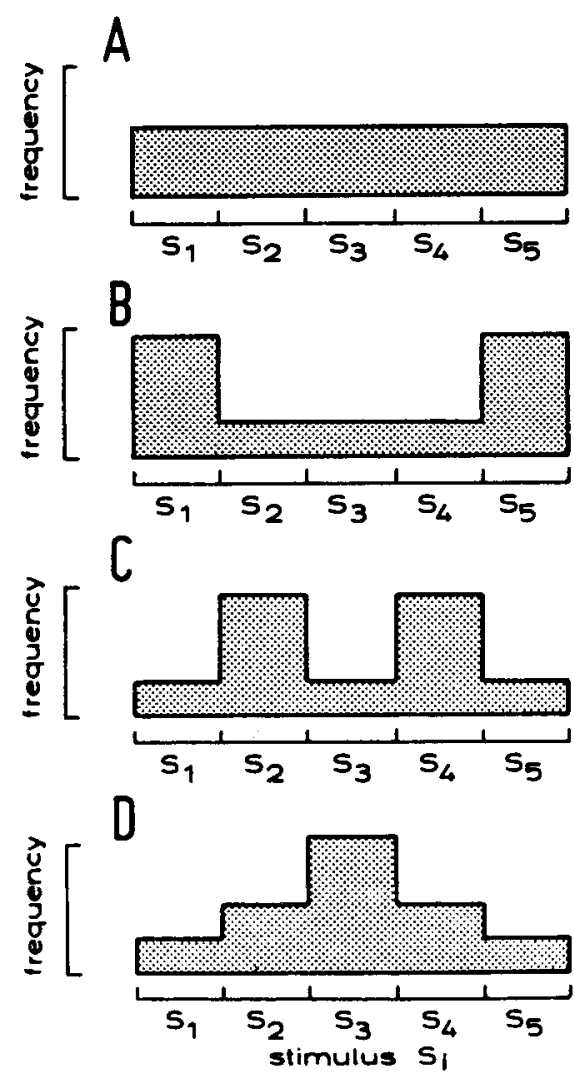

Figure 5. Frequency distribution of the stimulus sequences used in Experiment 7. Each sequence consisted of 80 trials. The height of the vertical bar corresponds to 32 trials. 
Table 1

Mean JNDs in Orientation $(N=2)$ for Experiments 5, 6, and 7

\begin{tabular}{|c|c|c|c|c|c|c|}
\hline $\begin{array}{c}\text { Experi- } \\
\text { ment }\end{array}$ & Subject & Standard* & \multicolumn{2}{|c|}{ Preview } & \multicolumn{2}{|c|}{ No Preview } \\
\hline \multirow[t]{5}{*}{5} & R.V. & $\mathrm{p}$ & \multicolumn{2}{|c|}{0.70} & \multicolumn{2}{|c|}{0.61} \\
\hline & & 0 & \multicolumn{2}{|c|}{1.90} & \multicolumn{2}{|c|}{2.08} \\
\hline & J.V. & $p$ & \multicolumn{2}{|c|}{1.13} & \multicolumn{2}{|c|}{1.03} \\
\hline & & o & \multicolumn{2}{|c|}{3.25} & \multicolumn{2}{|c|}{3.40} \\
\hline & & & \multicolumn{2}{|c|}{ Feedback } & \multicolumn{2}{|c|}{ No Feedback } \\
\hline \multirow[t]{6}{*}{6} & R.V. & p & \multicolumn{2}{|c|}{0.58} & \multicolumn{2}{|c|}{0.52} \\
\hline & & o & \multicolumn{2}{|c|}{2.10} & \multicolumn{2}{|c|}{1.38} \\
\hline & J.V. & p & \multicolumn{2}{|c|}{0.94} & \multicolumn{2}{|c|}{0.98} \\
\hline & & o & \multicolumn{2}{|c|}{2.90} & \multicolumn{2}{|c|}{2.35} \\
\hline & & & \multicolumn{4}{|c|}{$\begin{array}{c}\text { Frequency Distribution } \\
\text { of the Stimulus Set } \uparrow\end{array}$} \\
\hline & & & A & B & $\mathrm{C}$ & D \\
\hline \multirow[t]{4}{*}{7} & R.V. & $\mathrm{p}$ & $\overline{0.55}$ & 0.64 & 0.71 & 0.83 \\
\hline & & o & 1.35 & 1.59 & 1.79 & 1.29 \\
\hline & J.V. & $\mathrm{p}$ & 1.02 & 0.96 & 1.00 & 0.68 \\
\hline & & o & 3.15 & 1.98 & 2.02 & 2.27 \\
\hline
\end{tabular}

*p = principal standard orientation; $o=$ oblique standard orientation. †See Figure 5.

cal displacements of the functions measure the differences in criterion (or bias) due to sequential effects. In the ideal case, the slopes of these functions should be equal. The small differences in slope that we obtained could be due to stimulus-dependent changes in criterial noise (Treisman \& Faulkner, 1984). The magnitude of these differences in slope was similar at principal and oblique standard orientations: The median (for 3 subjects) standard deviation on the slope was 0.10 for the principal standard orientation and 0.09 for the oblique standard orientation.

In order to calculate a bias index that is independent of the differences in slope, we normalized the bias indices as follows: $C_{\mathrm{ni}}=C_{\mathrm{i}} \times(\max$ slope/slope $\mathrm{i})$. In this formula, $C_{\mathrm{ni}}$ stands for the normalized bias index for condition $\mathrm{i}, C_{\mathrm{i}}$ represents the $Z$ value of the criterion measured along the ordinate at $S_{i}=3$, max slope is the largest slope value of a set of slopes, and slope i represents the slope of condition $i$. The results of this normalization for the sets of slopes of Figure 6A are shown in Figure 6B. We performed all the calculations presented below on both the raw and the normalized bias indices. Since no systematic differences between those two analyses were found, we will present only the data for the normalized bias indices.

We plotted the normalized bias indices as a function of the preceding stimulus in Figure 7. Figure 7A shows the individual data; the results pooled over subjects are shown in Figure 7B. In these plots, a negative slope indicates a negative sequential dependency, or a contrast effect; a positive slope indicates a positive sequential dependency, or an assimilation effect. A negative sequential dependency is defined as a decrease in the probability of $R_{2}$ responses when $S_{5}$ was presented on the preceding trial. It implies a criterion shift toward the value of the preceding stimulus. In positive sequential dependencies, the probability of $\mathbf{R}_{\mathbf{2}}$ responses increases when the corresponding stimulus was presented on the preceding trial. For both standard orientations, a negative stimulus dependency, or contrast effect (Treisman \& Williams, 1984), is present.

These results were analyzed with a two-factorial block ANOVA (standard orientation $\times$ preceding stimulus). The negative sequential dependency proved to be significant $[F(1,18)=8.61, p<.01]$. However, the interaction between standard orientation and preceding stimulus was not significant $[F(4,18)<1$, n.s.]. We also calculated the

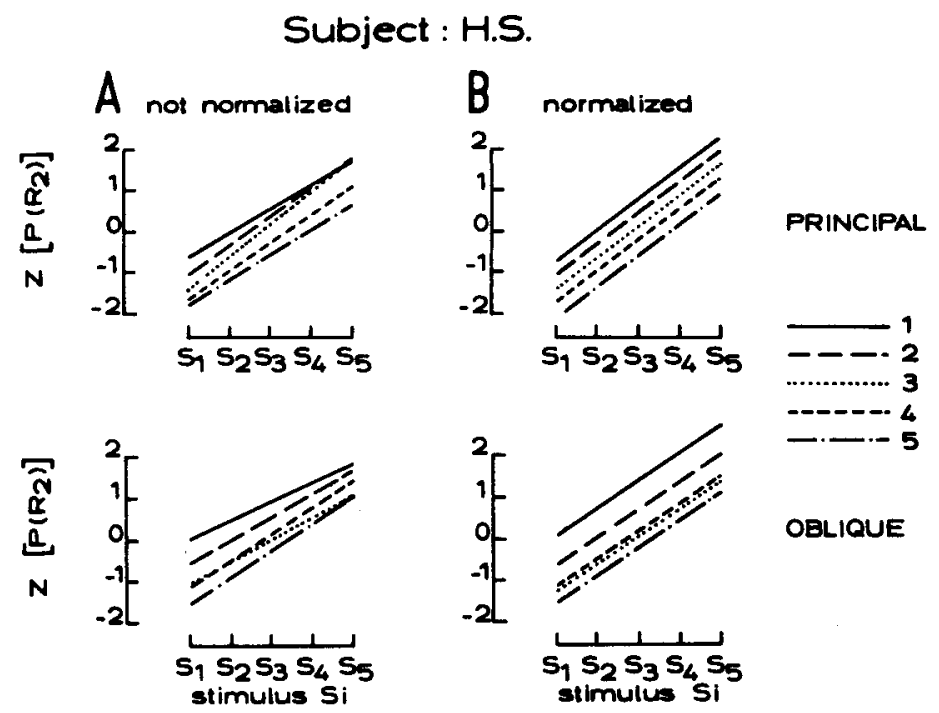

Figure 6. The function relating the normalized proportion of $R_{2}$ responses and the stimuli $S_{i}$ as a function of the preceding stimulus. The functions were obtained by linear regression. A. Not normalized functions. B. Normalized functions (see text). Preceding stimuli: $1: S_{1}, 2: S_{2}, 3: S_{3}, 4: S_{4}$, and $5: S_{3}$. Top: principal standard orientation. Bottom: oblique standard orientation. 


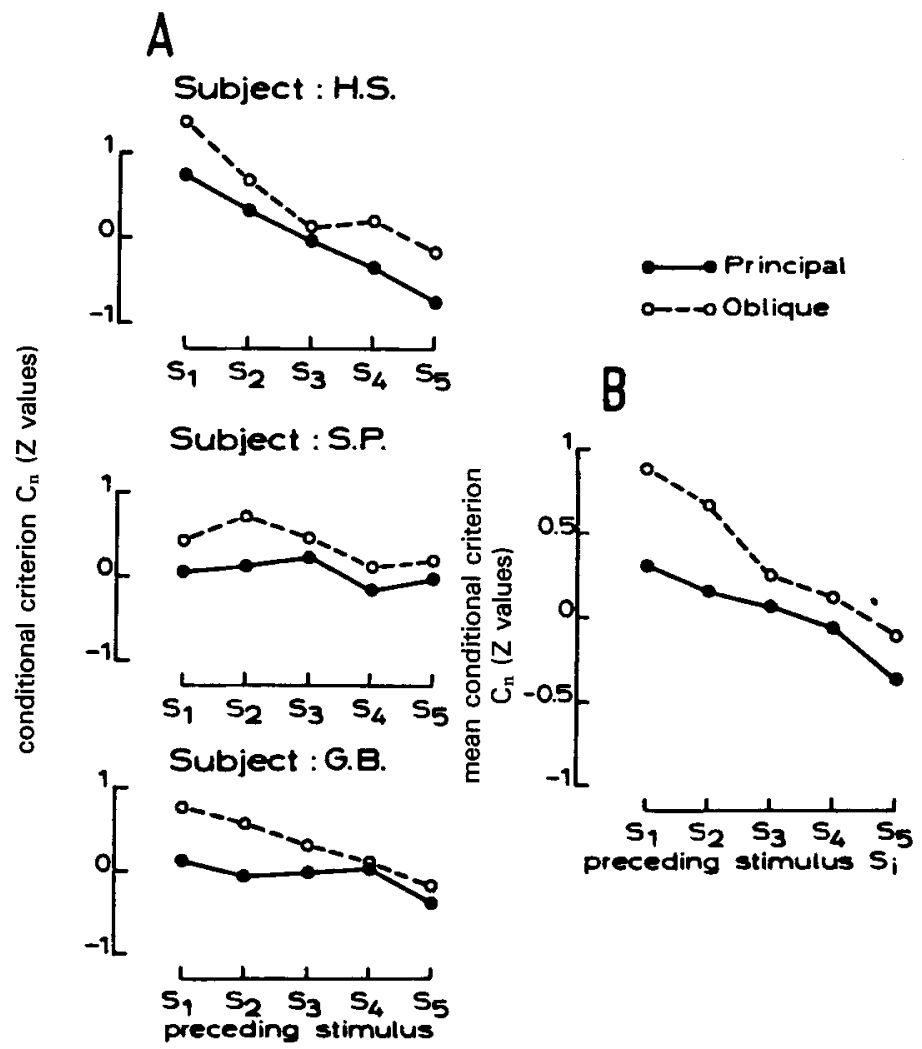

Figure 7. Stimulus-dependent sequential effects. The normalized bias index is plotted as a function of the preceding stimulus for the principal and oblique standard orientations. A. Individual data. B. Mean bias indices (for 3 subjects).

variance on the normalized bias indices at Lag 1 and at Lag 1 and Lag 2 combined for each orientation and subject. This yields an estimate of the criterial variance due to the stimulus-dependent sequential effects. However, we found no significant correlation of the criterial noise as estimated from the stimulus-dependent effects $\left(C_{\mathrm{ni}}\right)$ and the JND in orientation. The difference between JNDs at oblique and principal standard orientations is clearly too large to be explained by an anisotropy in stimulusdependent criterial changes.

The results on response dependencies are shown in Figure 8. In Figure 8A we plotted the normalized bias indices as a function of the preceding responses for different lags for 1 subject. These data can be condensed by calculating the difference in bias indices between the two responses. We plotted this difference in conditional bias as a function of the lag for each subject separately (Figure 8B) and averaged over subjects (Figure 8C). A positive difference corresponds to assimilation, whereas a negative difference indicates a negative sequential effect. At the horizontal standard orientation, a positive response dependency, or assimilation, is present (mainly for Lags 1 and 2); for the oblique standard orientation a negative effect is observed (only for Lag 1). This negative effect could be due to the negative stimulus-dependent effect (Figure 7), because, obviously, stimuli and responses are correlated. Subjects who show the strongest negative stimulus-dependent effect (H.S. and G.B.) also show a negative response-dependent effect at this standard orientation, whereas subject S.P., who shows a weaker negative stimulus-dependent effect, shows no negative response dependency (Figures 7A and 8B).

The sequential response-dependent effect seems to be more positive at the horizontal than at the oblique standard orientation. This interaction between standard orientation and preceding response was small but significant in an ANOVA $[F(1,22)=5.66, p<.05]$. However, because the average absolute difference between the conditional criteria is similar at oblique and principal standard orientations (Figure $8 \mathrm{C}$ ), the criterial noise due to response dependencies cannot explain the oblique effect in orientation discrimination.

\section{DISCUSSION}

Our results indicate that the oblique effect in orientation discrimination is not due to an anisotropy of the decision rule used in the MSS task. In fact, we demonstrated that when performing our MSS task, most subjects use an AI rule, and do so at all standard orientations: The difference in JND between stimulus sequences with different step sizes and between procedures with different num- 

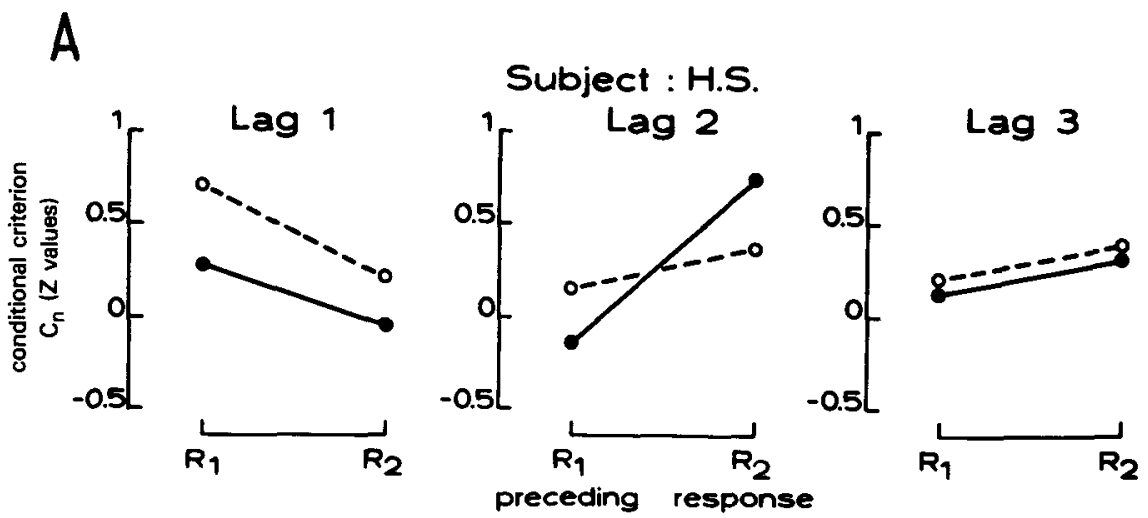

B

Subject : H.S.
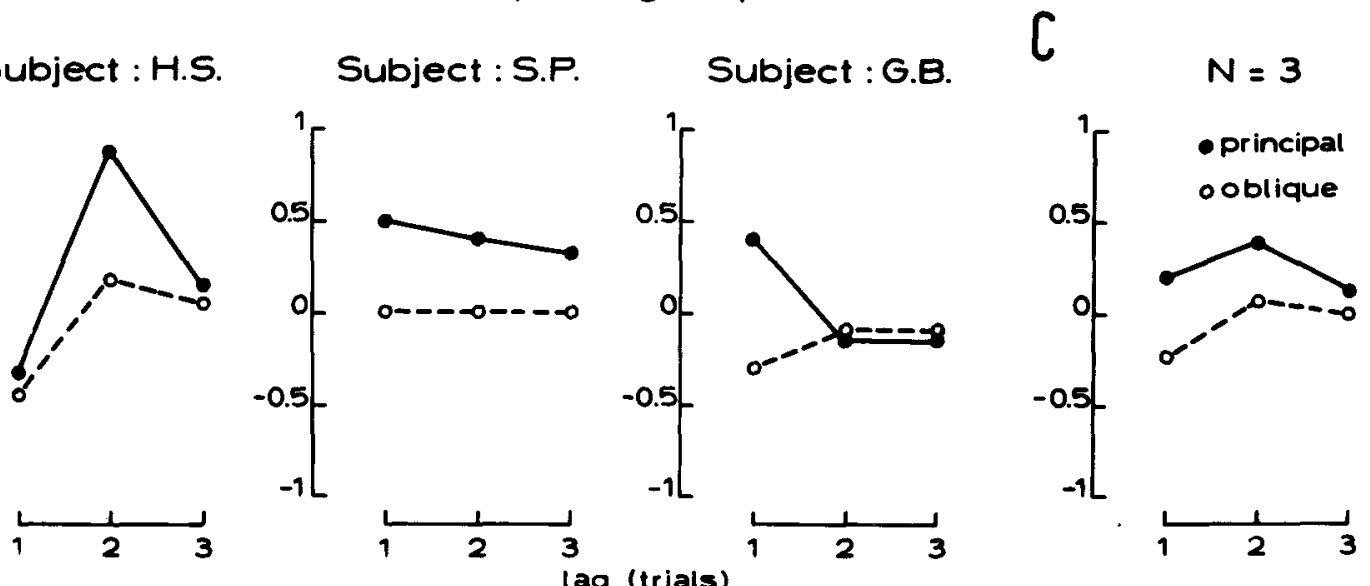

Figure 8. Response-dependent sequential effects. A. The normalized bias index at the principal (solid symbols) and oblique (open symbols) standard orientations is plotted as a function of the preceding response for three different lags. $B$. The difference between the bias indices of both preceding responses $\left(R_{1}\right.$ and $R_{2}$ in $\left.A\right)$ as a function of the lag for 3 subjects. C. Mean bias difference (for 3 subjects) due to the response dependencies as a function of the lag for the principal and oblique standard orientations.

bers of stimulus alternatives was much smaller than would be expected if subjects used a PC rule. Also, further analysis of the stimulus-dependent sequential effects supports this conclusion. If a subject used a PC rule, the distance measured at $Z=0$, between the regression lines corresponding to $S_{1}$ as preceding stimulus and $S_{5}$ as preceding stimulus in plots such as Figure 6, should equal the difference between $S_{1}$ and $S_{5}$. This results from the fact that under a $P C$ rule, $P\left(R_{2}\right)$ should be constant for all stimuli if the preceding stimulus and the stimulus of the present trial are the same. However, in none of our subjects was such a strong contrast effect observed. The meridional difference in the magnitude of the step size effect, unlike the oblique effect in the JNDs in orientation, was small, inconsistent, and largely individually variable. The same was true of the meridional difference in the negative stimulus-dependent sequential effect. In fact, the negative sequential stimulus dependency may explain the step size effect. A shift of the criterion toward the preceding stimulus will result in a higher proportion of incorrect responses to this stimulus or to neighboring stimuli on a later trial. Because the similarity between succeeding stimuli is much larger in sequences with a small step size than in sequences with a large step size, the proportion of correct responses will be smaller in the small step size condition.

Our results also suggest that in an MSS task the criterion is built up and maintained in the same way at principal and oblique standard orientations. This is difficult to explain if one assumes that the oblique effect is due to differences in criterial variance; a simple calculation shows that the criterial noise at the oblique orientations has to be much larger than the criterial noise at the principal orientations to explain the oblique effect. The results of such a calculation, using the data from 3 of our subjects, are shown in Figure 9. The criterial variance is expressed in sensorial variance units. The figure shows that the amount of criterial variance at the oblique standard orientations needed to explain the oblique effect strongly increases with increasing amount of the criterial variance at the principal orientations. Even if the criterial variance at principal orientations is extremely small, the criterial variance at the oblique orientations has to be at least a factor of 3 to 8 larger than the sensorial variance in order to explain the observed difference between the JNDs at the principal and oblique standard orientations. An analysis of the 


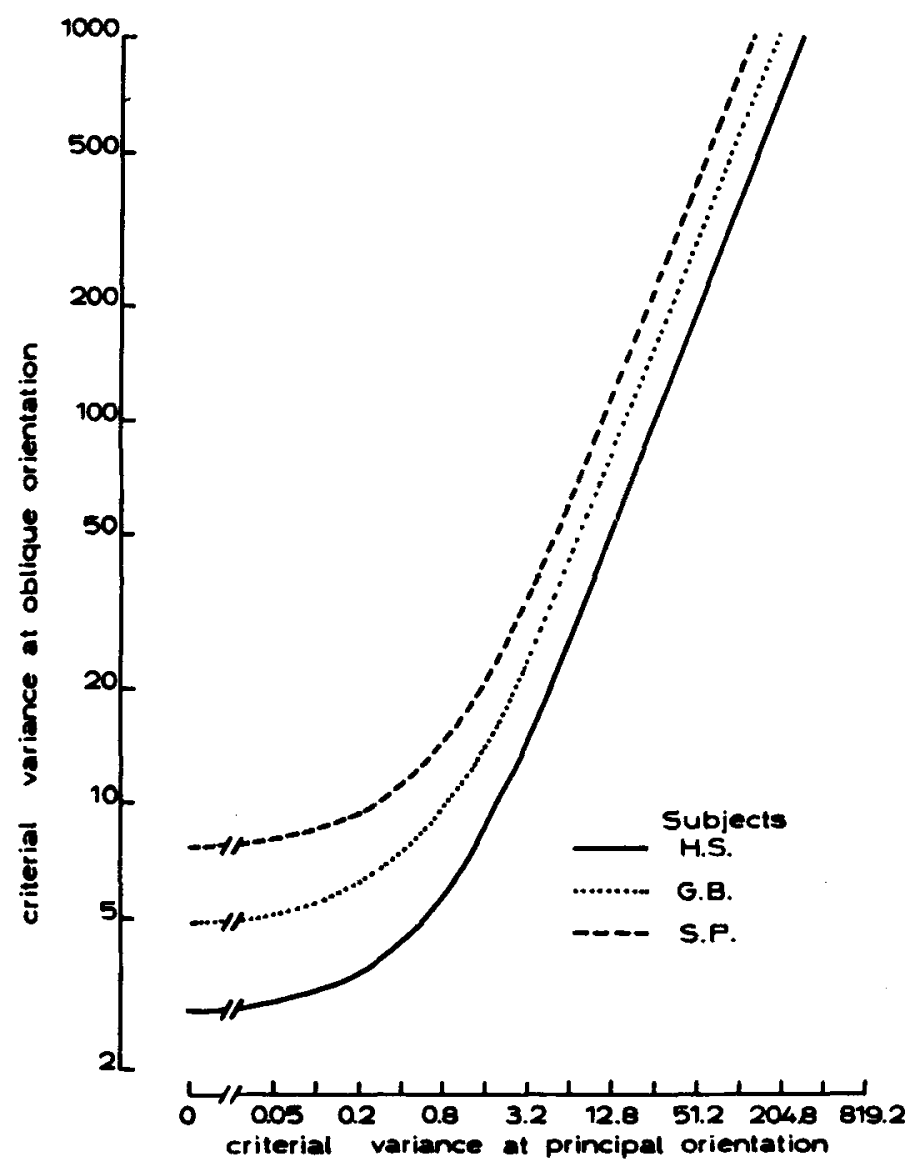

Figure 9. Criterial variance at the oblique orientations as a function of the criterial variance at the horizontal orientations. Both variances are expressed in sensorial variance units, which were equal for both orientations. The criterial variance at the oblique standard orientation was computed as a function of the amount of criterial variance at the horizontal standard orientation, assuming that the oblique effect observed in 3 subjects was due to a criterial variance anisotropy.

stimulus- and response-dependent criterion shifts also supports the conclusion that the oblique effect is not due to a larger criterial variance at the oblique orientations.

Hence these results strongly suggest that the oblique effect in line orientation discrimination as determined in an MSS task is not due to meridional variations in decision factors, but is the result of a sensorial anisotropy. The oblique effect is also found with other psychophysical methods, such as two-alternative forced-choice procedures, and it has been shown that, for these kinds of tasks as well, the effect is not due to decision factors (Vogels \& Orban, 1986). However, one must be cautious in generalizing this finding to absolute judgment tasks, because the oblique effect obtained with this method may be due at least partly to vocabulary factors (Matin et al., 1982) and to the use of the principal orientations as anchors. Although conditioning in labeling orientations may reduce the contribution of these response factors to performance in these absolute judgment tasks (Matin et al., 1982), the contribution of sensorially based anisotropies still remains to be determined.

In several experiments, the interaction between subject and the nonsensorial variable was significant. The magnitude of effect of step size, particularly, was strongly dependent on the subject (see Figure 2C). This suggests that some subjects have difficulty in building up or maintaining a stable criterion. This idea is supported by the large individual variation in sequential dependencies and hence in criterion maintenance. In a previous study (Vogels et al., 1984) we reported on the high individual variation in JNDs obtained with this MSS: At the principal standard orientations, JNDs differed over a five-fold range, and at the oblique standard orientation, JNDs spanned an eight-fold range. These individual variations in JND were positively correlated with the ratio (training-test ratio) between threshold estimates derived from the training step (an identification task) and the MSS JNDs. These training-test ratios varied from 0.5 to 4 
(Vogels et al., 1984). It is highly probable that this discrepancy in performance between training and test results from a high criterial variance due to weak criterial maintenance processes for a large number of stimulus alternatives. This could partly explain the large individual variation in JNDs obtained with the MSS procedure. However, these decision factors are probably not the only cause of the individual variations, inasmuch as individual differences that are similar but smaller in range are obtained with other psychophysical procedures (e.g., twoalternative forced-choice procedures) (Vogels \& Orban, 1986; Vogels et al., 1984).

Our results also provide some clues to the criterionsetting processes in MSS tasks in general. Recently, Treisman has developed a theory that links criterion-setting processes to sequential dependencies (for a detailed account, see Treisman \& Williams, 1984). According to this theory, criterion setting involves two stages-first, a general process of getting in range (building up a criterion), and second, short-term adjustments of this criterion by means of two systems, which can operate independently. The first system, called tracking, operates under the assumption that a stimulus persists. Therefore, it will enhance the difference between the current criterion and the stimulus in such a way that the probability of a future identification of the same stimulus increases. Hence, the tracking system induces positive sequential dependencies. The second, stabilizing, system uses the incoming information to position the criterion optimally and to keep it stable. This is done by moving the criterion in such a way that $\mathbf{P}\left(\mathbf{R}_{\mathbf{1}}\right)$ equals $\mathbf{P}\left(\mathbf{R}_{\mathbf{2}}\right)$. This will result in negative sequential dependencies, and largely in negative stimulus dependencies, since the difference between the current criterion and the stimulus provides information about the optimality of the current criterion (see Treisman \& Williams, 1984). We observed a strong negative stimulus dependency, which suggests that a stabilizing system, as hypothesized by Treisman and Williams, is operating to maintain the criterion. Our results indicate that the first-stage (getting in range) process is very fast (Experiments 3 and 4), and that a short-term stabilling mechanism is operating continuously to adjust the criterion (Experiment 4). That this adjustment or maintenance process is a short-term one is indicated by the quick reaction to a change in mean step size (Experiment 4). We found a small positive response dependency, which suggests that the tracking system plays only a minor role in the criterion setting in our MSS task. This is not surprising; a tracking system would perform suboptimally in tasks in which several stimuli are presented (see definition of tracking system).

To summarize, our results show that the oblique effect is due to a sensorially based anisotropy, and they therefore support the hypothesis linking the S-cell orientation anisotropy and the oblique effect in line orientation discrimination.

\section{REFERENCES}

Green, D. M., SWets, J. A. (1966). Signal detection theory and psychophysics. New York: Wiley.

Kennedy, H., Martin, K.A.C., Orban, G. A., \& Whitteridge, D. (1985). Receptive field properties of neurones in visual area 1 and visual area 2 in the baboon. Neuroscience, 14, 405-415.

KIRK, R. (1968). Experimental design: Procedures for the behavioral sciences. Belmont, CA: Brooks Cole.

Matin, E., DrIvas, A. (1979). Acuity for orientation measured with a sequential recognition task and signal detection methods. Perception \& Psychophysics, 25, 161-168.

Matin, E., Druvas, A., \& Valle, V. (1982). Conditioned tilt naming: A modified absolute judgment method is used to measure the oblique effect. Perception \& Psychophysics, 31, 421-428.

Orban, G. A., KenNEDY, H. (1981). The influence of eccentricity on receptive field types and orientation selectivity in areas 17 and 18 of the cat. Brain Research, 208, 203-208.

Orban, G. A., Vandenbussche, E., Vogels, R. (1984). Human orientation discrimination tested with long stimuli. Vision Research, 24, 121-128.

Treisman, M., \& FAUlKner, A. (1984). The setting and maintenance of criteria representing levels of confidence. Journal of Experimental Pyychology: Human Perception \& Performance, 10, 119-139.

Treisman, M., Williams, T. C. (1984). A theory of criterion setting with an application to sequential dependencies. Psychological Review, 91, 68-111.

Vandenbussche, E., Vogels, R., \& Orban, G. A. (1986). Human orientation discrimination: Changes with eccentricity in normal and amblyopic vision. Investigative Ophthalmology \& Visual Science, 27, 237-245.

VoGELS, R., \& ORBAN, G. A. (1986). Decision processes in visual discrimination of line orientation. Joumal of Experimental Psychology: Human Perception \& Performance, 12, 115-132.

Vogels, R., Orban, G. A., \& VANDEnbussche, E. (1984). Meridional variations in orientation discrimination in normal and amblyopic vision. Investigative Ophthalmology \& Visual Science, 25, 720-728.

(Manuscript received September 30, 1985; revision accepted for publication May 6, 1986.) 